\title{
Persian Version of the Yale Food Addiction Scale 2.0: Psychometric Analysis and Setting Cutoff Point for the Food Cravings Questionnaire-Trait-Reduced
}

\author{
Hourvash Haghighinejad ${ }^{1}$, Minoo Tarakemehzadeh², Peyman Jafari ${ }^{3}$, \\ Mahtab Jafari ${ }^{2}$, Mani Ramzi ${ }^{4}$, and Arvin Hedayati ${ }^{\circledR}$ \\ 'Department of Family Medicine, Shiraz University of Medical Sciences, Shiraz, Iran \\ ${ }^{2}$ Shiraz University of Medical Sciences, Shiraz, Iran \\ ${ }^{3}$ Department of Biostatistics, Shiraz University of Medical Sciences, Shiraz, Iran \\ ${ }^{4}$ Hematology Research Center, Shiraz University of Medical Sciences, Shiraz, Iran \\ ${ }^{5}$ Department of Psychiatry, Shiraz University of Medical Sciences, Shiraz, Iran
}

Objective The aims of this study were to evaluate the psychometric properties of Persian translation of the Yale Food Addiction Scale 2.0 (YFAS 2.0) as a widely accepted questionnaire for the first time and to establish a cut off score for Food Craving Questionnaire-Trait-reduced (FCQ-T-r).

Methods In this cross-sectional study, 330 visitors of family physician clinics in Shiraz, a city located in south of Iran, were selected. The English version of YFAS 2.0 was translated into Persian and used in this study as well as the Persian version of FCQ-T-r.

Results Confirmatory factor analysis of YFAS-2 confirmed one dimensional structure and factor loading in all eleven symptoms was above 0.4. Internal consistency for eleven symptoms was 0.813 . Prevalence of food addiction in participants was $6.7 \%$ (22 participants). BMI and FCQ-T-r questionnaire score both were positively correlated with the number of food addiction symptoms but age was negatively correlated with the number of the symptoms. The ROC curve analysis showed the best suggested cut-off point for FCQ-T-r questionnaire to detect food addiction was 32.5 .

Conclusion The present study confirmed validity and reliability of Persian version of YFAS-2. It is suggested that food addiction occurs in different level of food craving behavior in different food cultures or genetics. Psychiatry Investig 2021;18(3):179-186

Key Words Food addiction, Food craving, Yale Food Addiction Scale 2.0, Food Cravings Questionnaires Trait-reduced.

\section{INTRODUCTION}

Food addiction is an emerging term refers to overeating of palatable (such as high fat and high sugar) foods. It is a behavioral addiction which works through brain rewarding system. ${ }^{1,2}$

Randolph was the first researcher who defines food addiction as "a specific adaptation to one or more regularly consumed foods to which a person is highly sensitive, produces a common pattern of symptoms descriptively similar to those

Received: June 4, 2020 Revised: August 25, 2020

Accepted: October 16, 2020

$\triangle$ Correspondence: Arvin Hedayati, MD

Department of Psychiatry, Shiraz University of Medical Sciences, Hafez Hospital, Chamran Blvd., Shiraz, 7194634786 Iran

Tel: +98 713647 4967, Fax: +98 713647 4967, E-mail: hedayatia@sums.ac.ir

(c) This is an Open Access article distributed under the terms of the Creative Commons Attribution Non-Commercial License (https://creativecommons.org/licenses/bync/4.0) which permits unrestricted non-commercial use, distribution, and reproduction in any medium, provided the original work is properly cited. of other addictive processes." Addictive consumption patterns of foods were reported about some foods such as corn, wheat, milk, coffee, eggs, and potatoes. ${ }^{3}$

The increasing prevalence of obesity in the last two decades creates the idea that genetic factor alone cannot explain all this changes; instead environmental and behavioral factors must have an important role in making obesity a widespread problem. Food addiction can be a good explanation for overweight pandemic in the modern "obesogenic" environment which is characterized by availability of palatable, energy-dense and inexpensive foods. ${ }^{4}$

The results of brain imaging of obese people as well as people with substance dependence show functional similarities. ${ }^{1}$ Also, studies on animal models have shown addictive behaviors and brain changes associated with the consumption of sugar and high-calorie foods in mice.,

Some studies claim that food addiction is a misnomer and 
eating addiction must be replaced. They explain that food addiction which is a substance related phenome, is an ambiguous concept and overeating is a better phrase because it emphasizes on behavioral addiction to eating. ${ }^{7}$

Irrespective of motivating factors, chronic overeating leads to an uncontrollable increase in food intake that is resistant to change and treatment, and eventually in some susceptible nature, this behavior resembles substance abuse. ${ }^{4}$

Yale Food Addiction Scale (YFAS) was the first standardized scale which was developed for assessment of food addiction behavior based on diagnostic criteria for substance addiction in Diagnostic and Statistical Manual of Mental Disorders (DSMIV). ${ }^{8}$ With promotion of substance addiction criteria in newest version of DSM-5, Yale scale 2 revised recently to cover criteria changes. It also differs from original version in response options and some phrases.

The result of food addition assessment by old version of Yale food addiction shows that the mean prevalence of food addiction in the adult population of developed country was $19.9 \%$. The prevalence was higher in overweight and obese population, females and adults older than 35 years. ${ }^{10}$

One study showed that those who met the criteria of food addiction by YFAS, associated with lower scores in the physical, mental, and emotional dimensions of quality of life. ${ }^{11}$ People with diagnosis of Food addiction had a higher rate of depression than others. ${ }^{12}$

Obesity as a complication of food addiction is also associated with low social function in youth and children and reduced quality of life, ${ }^{13}$ emotional communication and interpersonal relationship ${ }^{14,15}$ and increased risk of cardiovascular disease, diabetes, hypertension, and some cancers. ${ }^{16}$

Base on validity studies of YFAS 2.0, BMI and food cravings have a positive correlation with food addiction. ${ }^{9,17}$

In fact, craving is one of the symptoms of addiction, which is associated with a worse prognosis. It is also true for food addiction. ${ }^{18-20}$ Food craving refers to a strong desire to consume a particular food. ${ }^{21,22}$ It is a very common phenomenon and about $100 \%$ of young women and $70 \%$ of young men have experienced this tendency at least once in the past year. ${ }^{23,24}$

Repetition of craving has shown a positive correlation with increased food intake and increased BMI. ${ }^{25}$ It has been shown those who diagnosed to be food addicted experience more intense and more frequent food craving. ${ }^{26}$

The Food Cravings Questionnaires (FCQs) are one of the most widely used measures for the assessment of food cravings. ${ }^{27}$ This questionnaire is not substance related and asks about craving in foods in general. Recently a 15 items short form of Food Cravings Questionnaires (FCQs), the FCQ Traitreduced (FCQ-T-r) have been developed which has a good internal consistency and reliability. Setting a cut off point for this measure was study by Meule. ${ }^{28} \mathrm{He}$ used Yale Food Addiction Scale 2.0 to set a cut off point for pathologic food craving. According to Meule's study a cutoff point of 50 on FCQ-T-r can distinguish between patients with and without food craving in Germany. ${ }^{28}$

Therefore, it is important to diagnose and have an appropriate approach to food addiction as a risk factor for obesity. Also, food craving as a very effective factor in food addiction can affect the therapeutic approach to this disorder. Therefore, understanding the relationship between these two disorders in different situations can be helpful in this regard.

The first aim of this study was to assess the internal structure expected for YFAS 2.0 and the psychometric properties of Persian translation of the YFAS 2.0 for the first time. On the other hand, because it is assumed that many factors such as the type of food intake are effective in development of food dependence, the other purpose of this study is to investigate the relationship between food addiction and food craving in a society with different food behaviors from Western societies. So as the second aim, the study investigate the expected correlation between food craving and food addiction assessed by FCQ-T-r andYFAS 2 questionnaires respectively in an eastern country.

\section{METHODS}

In this cross-sectional study, after receiving approval from the ethical committee of Shiraz University of Medical Sciences, samples were selected by cluster random sampling from visitors of family physician clinics in Shiraz, a city located in south of Iran.

To determine the sample size, 7 persons per each item of Yale Food Addiction Scale 2.0 were considered. Given the number of questions in the Yale 2.0, which was 35, the sample size calculated to be 245 . Because multistage and cluster sampling was used, the number multiplied by design effect 1.3 and final sample size was calculated to be 330 . There are 2 major health centers in Shiraz. From each of these centers 4 large health facilities each covered about 50000 citizens were randomly selected. According to population covered by each of these centers proportional convenient sampling was done from clients who referred to their family physicians.

Inclusion criteria was Age over 18 years old, willingness to participate in the study, be literate and understand the meaning of the questionnaire, spend at least 15 minutes to complete the questionnaire. The people who had visual difficulty were excluded from the study. Also, the questionnaire was read to the illiterate by the researcher and the answers were marked.

After obtaining written informed consent from individuals who agree to participate in the study, they were asked to com- 
plete data collection form, Yale Food Addiction Scale 2.0 and FCQ-T-r.

\section{Measures}

\section{YFAS 2.0.}

The scale is a 35 item self-administer questionnaire which assess certain food addiction over previous 12 months. Each item is scored on an 8 points scale range from 0 (never) to 7 (everyday). Each item has a different threshold, and if the score of that item is equal or above that threshold, that question is considered to be positive. ${ }^{9}$

Each of the 11 symptoms in Substance Related and Addictive Disorders proposed in the DSM- 5 will be assessed by two or more questions in the measure, and each diagnostic symptom is positive if the sum of the score of questions for that symptom is more than one. Finally, the questionnaire score calculated by adding up all symptoms endorsed, thus the final score will range between zero and eleven. In addition, based on the DSM-5 threshold for diagnosis of substance use disorder, different levels of severity can be distinguished: mild food addiction (when two or three criteria or symptoms are met), moderate food addiction (when four or five criteria are met), and Severe food addiction (when six or more criteria are met). The clinical impairment score is not considered in the scoring of the questionnaire but diagnosis of "food addiction" at all levels requires presence of clinical significant impairment due to eating disorder.

The English version of Yale Food Addiction Scale 2.0 was first translated into Persian by a fluent English and Persian speaker. Then the original and translated questionnaires were presented to five psychiatrists and psychologists of Shiraz University of Medical Sciences to confirm its content validity.

To measure the content validity index (CVI), the "relevance," "clarity" and "simplicity" of each item were determined by 4point Likert scale. For determining content validity ratio, the specialists were requested to score each item base on 3 point Likert scale (necessary, useful but not necessary and not necessary).$^{29}$ Content validity index and validity ratio were estimated and finally the indexes reached an acceptable level after multiple revisions. Then the Persian questionnaire was translated to English by a person with master's degree in English. There was no significance difference with original questionnaire.

\section{Food Cravings Questionnaire-Trait-reduced (FCQ-T-r)}

FCQ-T-r is a shorten version of original FCQ-T questionnaire which used 15 more relevant items from original scale. Validity and reliability of Persian version of the Food Cravings Questionnaire-Trait were confirmed by kachooei. Cronbach's alpha was 0.76 to 0.96 and test-retest reliability was 0.76 to $0.86 .^{30}$

Each item is scored on a 6 points scale range from 1 (never/ not applicable) to 6 (always). Higher score means more severe food craving. The final score is obtained from the sum of the scores for each question, which can range from 15 to 90 .

\section{Data analysis}

Confirmatory factor analysis was performed for dichotomous data by using Mplus software to evaluate if eleven symptoms of YFAS-2 have one dimensional structure. It must be mention that a sum score for each item of questionnaire is not used for final scoring. The score of YFAS-2 questionnaire was calculated based on eleven symptoms of addiction and a cutoff point was used to find if a symptom is met or not. Therefore, structural validity and reliability calculated based on symptoms not on each item score. Items which evaluated clinical significance impairment were not added to the score, ${ }^{9}$ therefore they were not included in this analysis.

Values of Comparative Fit Index and Tucker-Lewis Index more than 0.90, and Root Mean Square Error below 0.08 were considered acceptable model fit. ${ }^{31}$

Kuder-Richardson's $\alpha$ was used to assess internal consistency of the eleven symptoms. Other statistical analyzes were performed using SPSS 20 software. Spearman's correlation test was used to assess association between the number of YFAS food addiction symptoms and each of these characteristics and score: BMI, age and FCQ-T-r score. Independent ttest was used to compare age, BMI, score and number of food addiction symptoms in two groups with and without food addiction. To compare gender, marital status and educational level in these two groups, chi-square test was used. A p value $<0.05$ was considered to be significant.

Also, receiver-operating-characteristic (ROC) curve was used for estimating cut off point to distinguish between individuals with and without "food addiction" by Food Cravings Questionnaire-Trait-reduced.

\section{Ethical issues}

Approval for this study was given by the Ethics Committee of Shiraz University of Medical Sciences with ethical code: IR.sums.med.rec. 1398.82 .

\section{RESULTS}

Totally 330 individuals referred to family physician clinics participated in the study. The mean age of participants was 33.3 (SD: 10.3 , range: $18-73$ ). Overall, $65.2 \%$ of participants were women. 


\section{Questionnaire validity and reliability}

Content validity ratio and content validity index for all questions were equal to or more than $95 \%$ and $80 \%$ respectively which indicate acceptable content validity of the questionnaire.

The results of confirmatory factor analysis of YFAS-2 showed that factor loading in all eleven symptoms were acceptable (above 0.4) and Comparative Fit Index, Tucker-Lewis Index and Root Mean Square Error of Approximation all confirmed one dimensional structure. Internal consistency for eleven symptoms was 0.813 (Table 1 ).

Prevalence of food addiction in participants was 6.7\% (22 participants): $2.7 \%$ (9 participants) were classified as severe, $1.2 \%$ (4 participants) as moderate and $2.7 \%$ as mild food addiction. In term of age, sex and education level and marital status there was no significant difference between the two groups with and without food addiction. BMI and FCQ-T-r questionnaire score both were positively correlated with the number of food addiction symptoms and age was negatively correlated with the number of the symptoms (Table 2).

Figure 1 shows that withdrawal (59.1\%) and attempt to reduce or quit (54.5\%) was the most prevalent symptoms in people with food addiction and failure to fulfill obligations (4.5\%) was the lowest prevalent symptom. The prevalence of all eleven criteria was significantly different in the two groups with and without food addiction except for failure to fulfill obligations which was approximately equal in the two groups.

To investigate the second aim of the study, the relationship

Table 1. Factor loading of YFAS-2 symptoms and result of confirmatory factor analysis

\begin{tabular}{|c|c|}
\hline Symptoms & Standardized factor loading (SE) \\
\hline Symptom 1: amount & $0.731(0.048)$ \\
\hline Symptom 2: attempt & $0.861(0.069)$ \\
\hline Symptom 3: time & $0.802(0.101)$ \\
\hline Symptom 4: give up activity & $0.607(0.035)$ \\
\hline $\begin{array}{l}\text { Symptom 5: knowledge } \\
\text { of consequence }\end{array}$ & $0.947(0.052)$ \\
\hline Symptom 6: tolerance & $0.903(0.065)$ \\
\hline Symptom 7: withdrawal & $0.767(0.059)$ \\
\hline $\begin{array}{l}\text { Symptom 8: continued } \\
\text { use despite problems }\end{array}$ & $0.768(0.135)$ \\
\hline $\begin{array}{l}\text { Symptom 9: failure to fulfill } \\
\text { obligation }\end{array}$ & $0.426(0.062)$ \\
\hline $\begin{array}{l}\text { Symptom 10: physically } \\
\text { hazardous }\end{array}$ & $0.774(0.051)$ \\
\hline Symptom 11: craving & $0.895(0.048)$ \\
\hline \multirow[t]{2}{*}{ Goodness of fitness } & CFI TIL RMSEA $\chi^{2}$ (df) \\
\hline & $0.97 \quad 0.96$ \\
\hline
\end{tabular}

CFI: Comparative Fit Index, TLI: Tucker-Lewis Index, RMSEA: Root Mean Square Error of Approximation, YFAS-2: Yale Food Addiction Scale 2.0

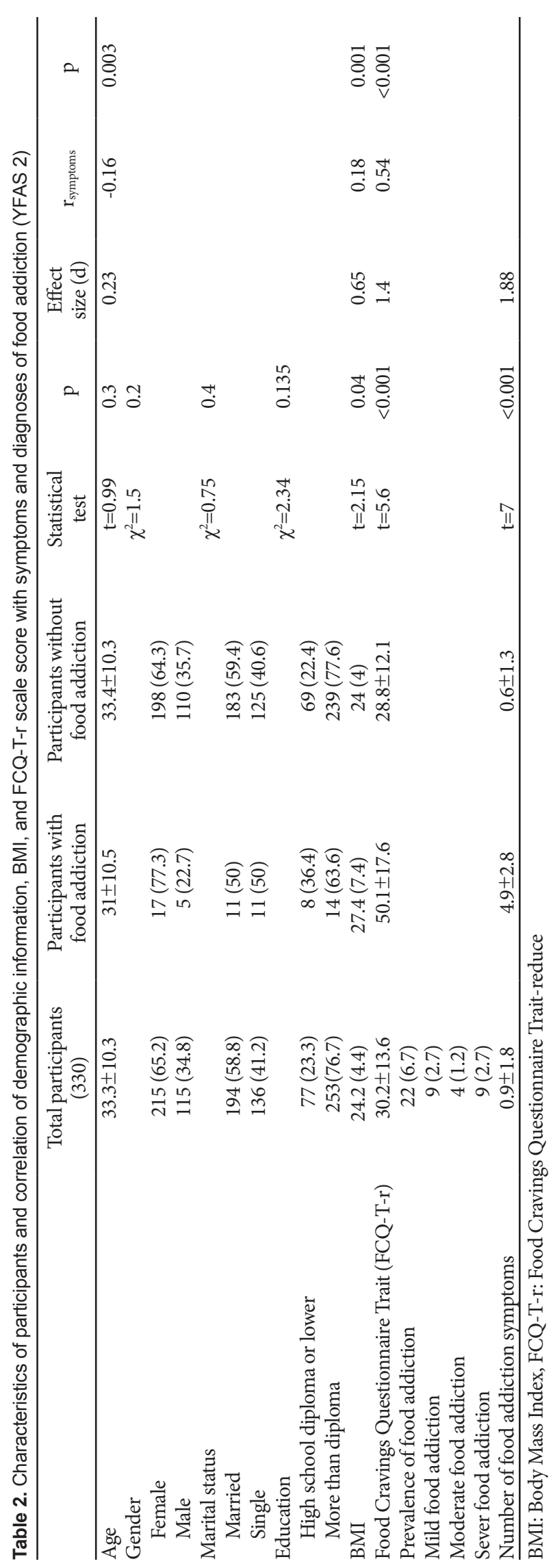

182 Psychiatry Investig 2021;18(3):179-186 
between diagnosis of food addiction (by YFAS-2) and food craving score (by FCQ-T-r) was evaluated. As it was shown in Figure 2 the frequency of higher score was higher in food addicted participants than those without food addiction.

Receiver-operating-characteristic curve analysis was performed to determine the cutoff point for the FCQ-T-r questionnaire to discriminate food addiction. The area under the curve was 0.85 (Figure 3). The suggested cut-off point was 32.5 with a sensitivity of $91 \%$ and specificity of $72 \%$. However, the points between 32.5 and 37.5 have sensitivity and specificity above 70\% (Table 3, Figure 3).

\section{DISCUSSION}

Food addiction has recently received much attention because of its role in weight gain. Yale Food Addiction Scale was one of the most widely accepted questionnaires designed according to DSM-5 criteria to diagnose food addiction. The present study, aimed to assess the internal structure, validity

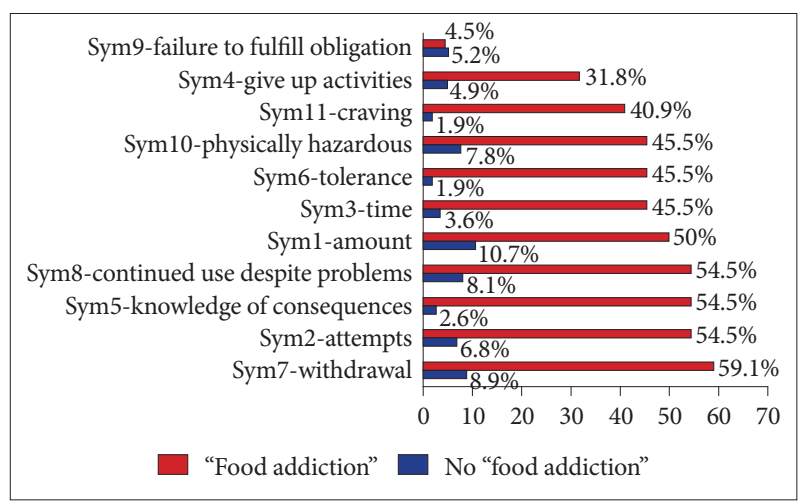

Figure 1. Frequency of the food addiction symptoms according to YFAS 2 scale. YFAS: Yale Food Addiction Scale.

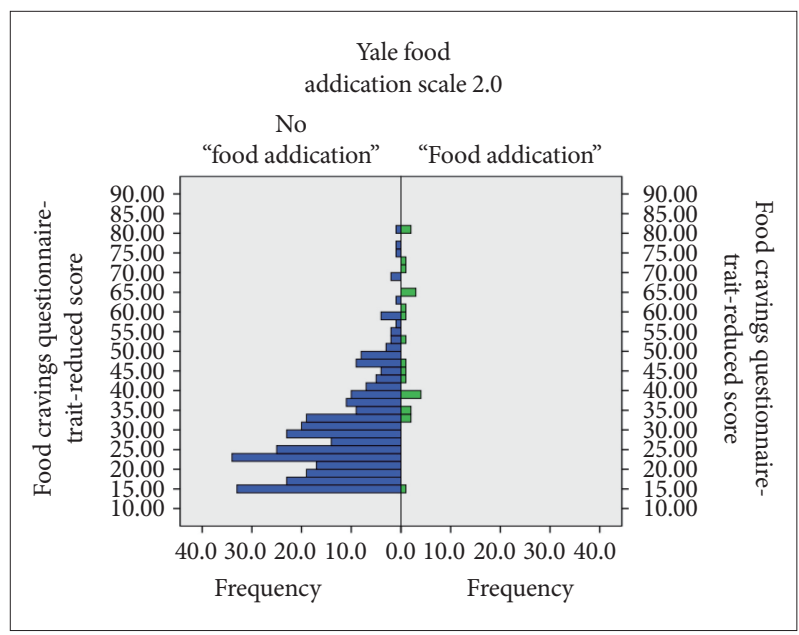

Figure 2. Comparison between two groups (no food addiction vs. food addiction classified by the Yale Food Addiction Scale 2.0) in term of frequencies of scores on food craving-trait-reduced questionnaire. and reliability of Persian version of Yale Food Addiction Scale 2.0. On the other hand, this study investigates the relationship between food addiction and food craving in a society with different food types and genetic from Western societies to assess the possible impact in this regard. The results confirmed unidimensional structure of Yale Food Addiction Scale 2.0. All eleven symptoms demonstrated the acceptable factor load. Also, it had been shown that the cut off point for detecting food addiction by food craving questionnaire (FCQ-T-r) is not entirely compatible with what is seen in Western societies.

Factor loadings for all the eleven symptoms, were greater than 0.4 in present study and the lowest factor loadings was for symptom 9 (failure to fulfill obligation), which was 0.42 . The

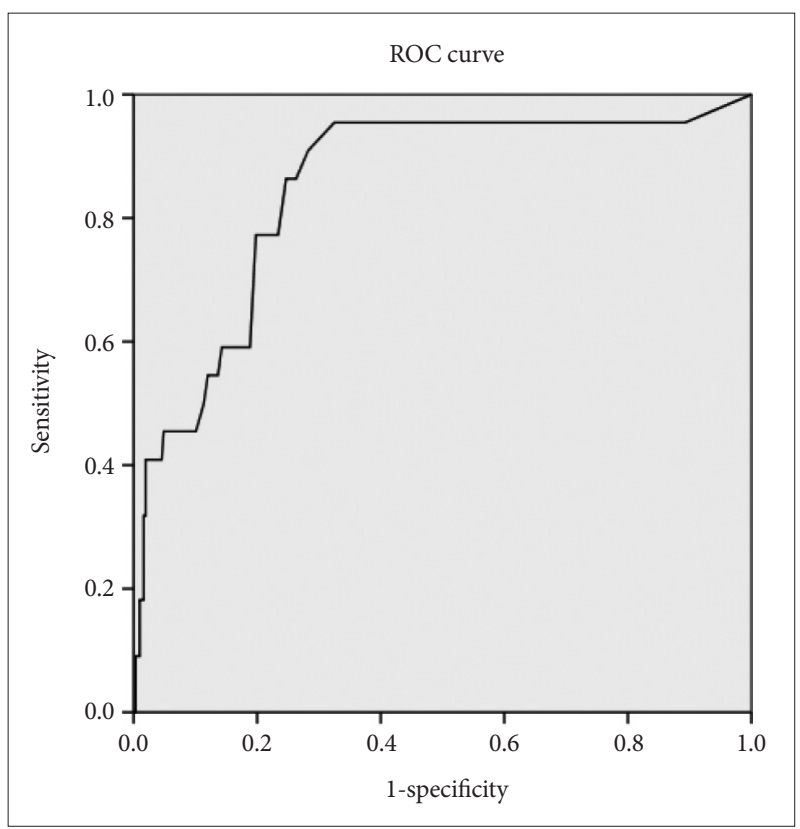

Figure 3. Receiver-operating-characteristic (ROC) curve for distinguishing between individuals with and without "food addiction" using the scores on Food Cravings Questionnaire-Trait-reduced.

Table 3. Sensitivity and specificity of different scores on the FCQT-r craving questionnaire to differentiate between individuals with and without "food addiction"

\begin{tabular}{lccc}
\hline \multicolumn{3}{c}{ Coordinates of the curve } \\
\hline Test result variable (s): \\
\hline FCQ-T-r score & Sensitivity & Specificity & $\begin{array}{c}\text { Sensitivity } \times \\
\text { Specificity }\end{array}$ \\
\hline 32.5000 & 0.909 & 0.718 & 0.652662 \\
33.5000 & 0.864 & 0.737 & 0.636768 \\
34.5000 & 0.864 & 0.753 & 0.650592 \\
35.5000 & 0.773 & 0.766 & 0.592118 \\
36.5000 & 0.773 & 0.776 & 0.599848 \\
37.5000 & 0.773 & 0.802 & 0.619946 \\
\hline
\end{tabular}

FCQ-T-r: Food Cravings Questionnaire-Trait-reduced 
results of comparative fit index (CFI: 0.97), Tucker-Lewis index (TLI: 0.96) and root mean square error of approximation (RMSEA: 0.047) confirm the suitability of the one-dimensional model. The internal consistency of the Yale-2 questionnaire for eleven symptoms was acceptable. The result is comparable with English, French, German, Portuguese, Italian and Japanese versions of the questionnaire which all confirmed it to be one dimensional scale. ${ }^{9,17,32-35}$

The results are also comparable with the study by Gearhardt et al. ${ }^{9}$ in 2016 which evaluate reliability and validity of the YFAS-2 for the first timewhich showed TLI: 0.974, CFI: 0.958, RMSEA: 0.108 with Kuder-Richardson's $\alpha$ of 0.9. ${ }^{9}$

The two symptoms of "withdrawal" and "attempt to reduce or quit" were the most prevalent in people with food addiction. Avoiding food or dietary restriction means losing food rewards that are very difficult to resist. ${ }^{36}$ Reward dependence explains an important part of the biological definitions of addiction ${ }^{37}$ so it is completely compatible with what the questionnaire was designed to detect. The criterion "failure to fulfill obligation" had the least prevalence among people with food addiction. The prevalence of all eleven criteria for food addiction was also significantly different between the two groups of people with and without food addiction, with the exception of "failure to fulfill obligation," which was approximately equal in the two groups.

In a 2016 study by Gearhardt et al., ${ }^{9}$ Two measures of "withdrawal" and "attempt to reduce or quit" had the highest prevalence in people with eating disorders, but the lowest prevalence of symptoms was related to "give up important activities." In the present study, also, give up important activities was the second less common symptom.

In the study of Meule et al., ${ }^{17}$ the criterion "failure to fulfill obligation" had also relatively low prevalence compare to other symptoms. In Japanese version of YFAS-2, the factor load of "failure in role obligation" was somewhat unacceptable $(<0.4){ }^{35}$ In the present study, this criterion had the lowest factor load among other symptoms, although its load was acceptable but it seems that the symptom was less distinctive in the diagnosis of food addiction or because of cultural issue, it is reported less by the participants.

Prevalence of the food addiction was $6.7 \%$ in the present study population. BMI and FCQ-T-r questionnaire score both were positively correlated with the number of food addiction symptoms and age was negatively correlated with the number of the symptoms. Also, a score of 32.5 in food craving questionnaire was identified for food addiction.

The prevalence of food addiction was acceptable compared to similar studies in France and Germany, with a prevalence of $8.7 \%$ and $8.8 \%$ respectively. ${ }^{32}$ Also it is comparable with Chinese study which the prevalence in diabetes and control group was about $8.6 \%$ and $1.3 \%$ respectively. ${ }^{38}$ In the United States the prevalence was $11.4 \%$ which was much higher than results of the present study, may be due to the high rate of overweight in the United States. ${ }^{32}$ a meta-analysis of developed country studies has estimated food addiction prevalence to be $19.9 \%$ (ranging from 5.4\% to 50\%). ${ }^{11}$ The higher prevalence in these studies compare to results of present study is partly due to differences in diet in industrialized countries compared to developing countries. People In industrialized countries; perhaps due to the higher consumption of processed, high-calorie foods, are more likely to develop food addiction.

In the present study, gender, education, and marital status were not significantly different between the two groups of food addicts and non-addicts. BMI and FCQ-T-r questionnaire score both were positively correlated with the number of food addiction symptoms but age was negatively correlated with the number of the symptoms. These results were consistent with Gearhardt study except for age which was not associated with symptoms of food addiction and, on average, women were significantly more likely to have food addiction symptoms than men, as the authors have stated it may be due to the larger number of women participated in the study. ${ }^{9}$

In a study on German students by Meule et al., ${ }^{17}$ the number of symptoms was positively correlated with age, FCQ-T-r score and BMI. These findings are consistent with the results of the present study, except for the relationship between age and number of symptoms. This difference may be partly due to the differences in the study population. In the Meule's study, Participants consist of younger students. Probably in this age group of the community, food addiction increases by aging. But the present study covers the age of $18-50$ years, and lesser prevalence of food addiction in older age group may be due to their lower exposure to a high-calorie diet (which is a result of industrialization) as the leading cause of food addiction at their younger age.

The mean score of FCQ-T-r in our study was 28.8 in no food addiction group and 50.1 for those with food addiction. In a similar study of German students, the mean score was 31.5 in no food addiction group and 61 for those with food addiction. All these data suggested that food addiction and food craving is less severe in our population than western population. ${ }^{28}$

On the other hand, in the present study, the cut-off point of 32.5 was suggested to detect food addiction by FCQ-T-r questionnaire with $91 \%$ sensitivity and $72 \%$ specificity. In similar study by Meule in Germany in 2018, the cut-off point of 50 (range between 32 and 54) was suggested for this questionnaire to detect food addiction. ${ }^{28}$

Although the result of the present study is within the range suggested by Meule's study but it is lower than best cut-off point they suggested. As shown in Figure 1, craving as a symp- 
tom of food addiction has much higher prevalence among people with addiction (40.9\%) than people without addiction (1.9\%). These results indicate that in the Iranian population and in people of different ages, food addiction occurs at an earlier stage of craving. This may be due to the differences in age of the two studies or the differences in cultural, eating habits or genetic in the two countries. It is likely that in our country, food addiction can occur in the early stages of craving because of the higher consumption of foods high in starch (rice and bread). In addition, since it is assumed that food addiction can have genetic predisposing factors, ${ }^{39}$ so it is also possible that genetic factors influence this relationship. This means that despite of positive correlation between food craving and food addiction, these are two independent concepts which have shown no fixed rigid correlation in different situations. Komatsu's study in Japan also supports the possible impact of food culture on food cravings. ${ }^{40}$ However, proving this hypothesis requires further investigation in the general population of different countries with a larger sample size. It is suggested that the level of food addiction occurs in different level of food craving behavior in different food cultures.

In a study done by Inamorati et al. ${ }^{41}$ in Italy, reached a cutoff point of 57.5 on FCQ-T-r score to differentiate between those with and without food addiction. The study of sensitivity and specificity was not too high (both 69\%). Perhaps one of the reasons for the high cut-off score in this study was that it differentiates between those with moderate and severe overeating.

Limitation of our study is that it was performed on a sample population that was randomly collected from Shiraz (one of the 5 largest cities in Iran). In terms of food types, this population can represent the majority of the urban population of the country, but it does not include the rural population, so it does not represent the total population of the country. Therefore, more extensive research with more sample size may be able to provide us with more information in the future. Also, self-reporting nature of YFAS-2 and items like BMI may cause some deviation from the truth.

Finally, the present study confirmed validity and reliability of Persian version of YFAS- 2 and provided preliminary evidence for determining the cut-off point on FCQ-T-r, which was positively correlated with food addiction. Therefore, further studies are needed to evaluate the usefulness of this cutoff point to identify people with food addiction.

\section{Acknowledgments}

The Vice Chancellor for Research at Shiraz University of Medical Sciences funded this project. This research was performed by Minoo Tarakemehzadeh in partial fulfillment of the requirements for certification as a general practitioner at Shiraz University of Medical Sciences in Shiraz, Iran with grant number: 97-01-01-16993.

\section{Conflicts of Interest}

The authors have no potential conflicts of interest to disclose.

\section{Author Contributions}

Conceptualization: Hourvash Haghighinejad, Arvin Hedayati. Data curation: Hourvash Haghighinejad, Minoo Tarakemehzadeh, Mahtab Jafari. Formal analysis: Hourvash Haghighinejad, Peyman Jafari. Funding acquisition: Hourvash Haghighinejad. Investigation: Hourvash Haghighinejad, Minoo Tarakemehzadeh, Mani Ramzi. Methodology: Hourvash Haghighinejad, Mani Ramzi. Project administration: Hourvash Haghighinejad, Mani Ramzi. Resources: Hourvash Haghighinejad. Software: Hourvash Haghighinejad, Peyman Jafari. Supervision: Hourvash Haghighinejad. Validation: all authors. Visualization: all authors. Writing-original draft: all authors. Writing-review \& editing: all authors.

\section{ORCID iDs}

Hourvash Haghighinejad

Minoo Tarakemehzadeh

Peyman Jafari

Mahtab Jafari

Mani Ramzi

Arvin Hedayati https://orcid.org/0000-0002-9923-5664 https://orcid.org/0000-0003-3350-1744 https://orcid.org/0000-0002-4957-887X https://orcid.org/0000-0003-4075-4192 https://orcid.org/0000-0003-2878-4559 https://orcid.org/0000-0002-7960-1452

\section{REFERENCES}

1. Volkow ND, Wise RA. How can drug addiction help us understand obesity? Nat Neurosci 2005;8:555-560.

2. Hoebel BG, Hernandez L, Schwartz DH, Mark GP, Hunter GA. Microdialysis studies of brain norepinephrine, serotonin, and dopamine release during ingestive behavior. Theoretical and clinical implications. Ann N Y Acad Sci 1989;575:171-193.

3. Randolph TG. The descriptive features of food addiction; addictive eating and drinking. Q J Stud Alcohol 1956;17:198-224.

4. Davis C. From passive overeating to "food addiction": a spectrum of compulsion and severity. ISRN Obesity 2013;2013(435027):1-20.

5. Avena NM, Rada P, Hoebel BG. Evidence for sugar addiction: behavioral and neurochemical effects of intermittent, excessive sugar intake. Neurosci Biobehav Rev 2008;32:20-39.

6. Johnson PM, Kenny PJ. Dopamine D2 receptors in addiction-like reward dysfunction and compulsive eating in obese rats. Nat Neurosci 2010;13:635-641.

7. Hebebrand J, Albayrak Ö, Adan R, Antel J, Dieguez C, de Jong J, et al. "Eating addiction", rather than "food addiction", better captures addictive-like eating behavior. Neurosci Biobehav Rev 2014;47:295-306.

8. Gearhardt AN, Corbin WR, Brownell KD. Preliminary validation of the Yale food addiction scale. Appetite 2009;52:430-436.

9. Gearhardt AN, Corbin WR, Brownell KD. Development of the Yale Food Addiction Scale Version 2.0. Psychol Addict Behav 2016;30:113121.

10. Pursey KM, Stanwell P, Gearhardt AN, Collins CE, Burrows TL. The prevalence of food addiction as assessed by the Yale Food Addiction Scale: a systematic review. Nutrients 2014;6:4552-4590.

11. Chao AM, Shaw JA, Pearl RL, Alamuddin N, Hopkins CM, Bakizada ZM, et al. Prevalence and psychosocial correlates of food addiction in persons with obesity seeking weight reduction. Compr Psychiatry 2017; 73:97-104.

12. Kiyici S, Koca N, Sigirli D, Aslan BB, Guclu M, Kisakol G. Food addiction correlates with psychosocial functioning more than metabolic parameters in patients with obesity. Metab Syndr Relat Disord 2020;18: 161-167.

13. Kjelgaard HH, Holstein BE, Due P, Brixval CS, Rasmussen M. Adolescent weight status: associations with structural and functional dimensions of social relations. J AdolescHealth 2017;60:460-468.

14. Puhl R, Brownell KD. Bias, discrimination, and obesity. Obes Res 2001; 
9:788-805.

15. Puhl RM, Heuer CA. The stigma of obesity: a review and update. Obesity 2009;17:941-964.

16. Bray GA. Medical consequences of obesity. J Clin Endocrinol Metab 2004;89:2583-2589.

17. Meule A, Müller A, Gearhardt AN, Blechert J. German version of the Yale Food Addiction Scale 2.0: prevalence and correlates of 'food addiction' in students and obese individuals. Appetite 2017;115:54-61.

18. Gendall KA, Sullivan PF, Joyce PR, Fear JL, Bulik CM. Psychopathology and personality of young women who experience food cravings. Addict Behav1997;22:545-555.

19. Meule A, Lutz A, Vögele C, Kübler A. Food cravings discriminate differentially between successful and unsuccessful dieters and non-dieters. Validation of the Food Cravings Questionnaires in German. Appetite 2012;58:88-97.

20. Meule A, Westenhöfer J, Kübler A. Food cravings mediate the relationship between rigid, but not flexible control of eating behavior and dieting success. Appetite 2011;57:582-584.

21. ekic M, McClelland J, Campbell I, Nestler S, Rubia K, David AS, et al. The effects of prefrontal cortex transcranial direct current stimulation (tDCS) on food craving and temporal discounting in women with frequent food cravings. Appetite 2014;78:55-62.

22. Weingarten HP, Elston D. The phenomenology of food cravings. Appetite 1990;15:231-246.

23. Pelchat ML. Food cravings in young and elderly adults. Appetite 1997;28: 103-113.

24. Lopez RB, Hofmann W, Wagner DD, Kelley WM, Heatherton TF. Neural predictors of giving in to temptation in daily life. Psychol Sci 2014;25: 1337-1344.

25. Hill AJ, Weaver CF, Blundell JE. Food craving, dietary restraint and mood. Appetite 1991;17:187-197.

26. Meule A, Hermann T, Kübler A. Food addiction in overweight and obese adolescents seeking weight-loss treatment. Eur Eat Disord Rev 2015;23:193-198.

27. Cepeda-Benito A, Gleaves DH, Williams TL, Erath SA. The development and validation of the state and trait food-cravings questionnaires. Behav Ther 2000;31:151-173.

28. Meule A. Food cravings in food addiction: exploring a potential cutoff value of the Food Cravings Questionnaire-Trait-reduced. Eat Weight Disord 2018;23:39-43.
29. Theoretical foundations of scaling and standardization. Available at: http://ssu.ac.ir/cms/fileadmin/user_upload/Daneshkadaha/dbehdasht/tose_amuzesh/mataleb_amoozeshi/workshop/marahel_sakht_ azmoon.docx Accessed May 30, 2020.

30. Kachooei M, Ashrafi E. Exploring the factor structure, reliability and validity of the Food Craving Questionnaire-Trait in Iranian adults. J Kerman Univ Med Sci 2016;23:631-648.

31. Browne M, Cudeck R. Alternative ways of assessing model fit. Sociol Methods Res 1992;21:230-258.

32. Brunault P, Ballon N, Gaillard P, Réveillère C, Courtois R. Validation of the French version of the Yale Food Addiction Scale: an examination of its factor structure, reliability, and construct validity in a nonclinical sample. Can J Psychiatry 2014;59:276-284.

33. Torres S, Camacho M, Costa P, Ribeiro G, Santos O, Vieira FM, et al. Psychometric properties of the Portuguese version of the Yale Food Addiction Scale. Eat Weight Disord 2017;22:259-267.

34. Aloi M, Rania M, Muñoz RC, Murcia SJ, Fernández-Aranda F, De Fazio P, Segura-Garcia C. Validation of the Italian version of the Yale Food Addiction Scale 2.0 (I-YFAS 2.0) in a sample of undergraduate students. Eat Weight Disord 2017;22:527-533.

35. Khine MT, Ota A, Gearhardt AN, Fujisawa A, Morita M, Minagawa A, et al. Validation of the Japanese version of the yale food addiction scale 2.0 (J-YFAS 2.0). Nutrients 2019;11:687.

36. Rogers PJ. Food and drug addictions: similarities and differences. Pharmacol Biochem Behav 2017;153:182-190.

37. Meule A. Focus: Addiction: back by popular demand: a narrative review on the history of food addiction research. Yale J Biol Med 2015;88:295302.

38. Yang F, Liu A, Li Y, Lai Y, Wang G, Sun C, et al. Food addiction in patients with newly diagnosed type 2 diabetes in Northeast China. Front Endocrinol 2017;8:218.

39. Davis C, Bonder R. Genetics and Epigenetics of Food Addiction. In: Cottone P, Sabino V, Moore CF, Koob GF, Editors. Compulsive Eating Behavior and Food Addiction. Boston, MA: Academic Press;2019, p.293-327.

40. Komatsu S. Rice and sushi cravings: a preliminary study of food craving among Japanese females. Appetite 2008;50:353-358.

41. Innamorati M, Imperatori C, Meule A, Lamis DA, Contardi A, Balsamo M, et al. Psychometric properties of the Italian food cravings questionnaire-trait-reduced (FCQ-Tr). Eat weight Disord 2015;20:129-135. 